\title{
Tonology and sentence structure in Greek
}

\author{
Antonis Botinis ${ }^{1}$, Athina Kontostavlaki ${ }^{1}$, Olga Nikolaenkova ${ }^{2}$, \\ Charalambos Themistocleous ${ }^{3,4}$ \\ ${ }^{1}$ Lab of Phonetics and Computational Linguistics, University of Athens, Greece \\ ${ }^{2}$ Department of General Linguistics, Saint Petersburg State University, Russia \\ ${ }^{3}$ Department of Neurology, Johns Hopkins University, USA \\ ${ }^{4}$ Department of Swedish, University of Gothenburg, Sweden \\ https://doi.org/10.36505/ExLing-2019/10/0007/000369
}

\begin{abstract}
This is an experimental investigation of tonal production as a function of lexical stress variability and sentence types in Greek. One production experiment was carried out according to which the lexical stress assignment in the last words varied in the last three syllables in the context of polylectic and monolectic statement and alternative (polar) question sentence contexts. The results indicate that statements are associated with a rise-fall tonal pattern encompassing the last stressed syllable whereas the questions are associated with a rise-fall tonal pattern encompassing the last syllable of the utterance. The initial rise of the rise-fall in statements may be trancated whereas the rise-fall in alternative questions remained fairly invariable.
\end{abstract}

Keywords: tonal production, sentence structure, prosody, phonetics, Greek

\section{Introduction}

This is part of a larger experimental investigation with regards to tonological structures as a function of lexical stress focus and sentence type variability. In the present paper, we will report the results of a qualitative analysis with reference to tonal patterns in variable lexical stress and sentence type contexts. The lexical stress is constrained by a three-syllable window in Greek, namely only ultimate, penultimate and antepenultimate syllables can be associated with stress. At the postlexical level, stress is manifested differently depending on the sentence types and variable focus and sentence types structures. Focus productions in declarative contexts are usually associated with a rise-fall expansion of the tonal range locally on the stressed syllable of the speech unit in focus and a tonal compression on the post focus speech material (Botinis 1989). Alternative questions, usually referred to as "total", "polar" and "yes/no" questions, are usually associated with a tonal rise-fall pattern at the right edge of the respective speech unit (Botinis, Chaida, Nikolaenkova, Nirgianaki 2016).

This study aims to show the distinct patterns of stress, number syllables and their effects with the distinct tonal patterns of questions and statements in Greek.

ExLing 2019: Proceedings of $10^{\text {th }}$ International Conference of Experimental Linguistics, 25-27 September 2019, Lisbon, Portugal 


\section{Methodology}

The experimental material was recorded in a soundproof room at the University of Athens. A Zoom $\mathrm{H} 4 \mathrm{n}$ audio recorder was used, and the recordings were coded as single mono sounds (sampling frequency: $22050 \mathrm{~Hz}$ ) and transferred to a PC for further analysis using a compact SD card. Tokens were typed in Standard Greek orthography. Test materials were manually segmented and labelled by using simultaneous inspections of waveforms and wide-band spectrograms (see Figures 1-2).

\section{Results}

The results of the present investigation are show in figures 1-2. Figure 1 shows the production of the Subject-Verb-Prepositional Phrase sentence. Each production has a different stress pattern associated with the final word, namely the stress is in the antepenultimate (Figure 1, upper left and right panel), the penultimate (Figure 1, left and right middle panel) and ultimate (Figure 1lower left and right panels). All left panels depict statements and all right panel depict Wh. Question tonal patterns.

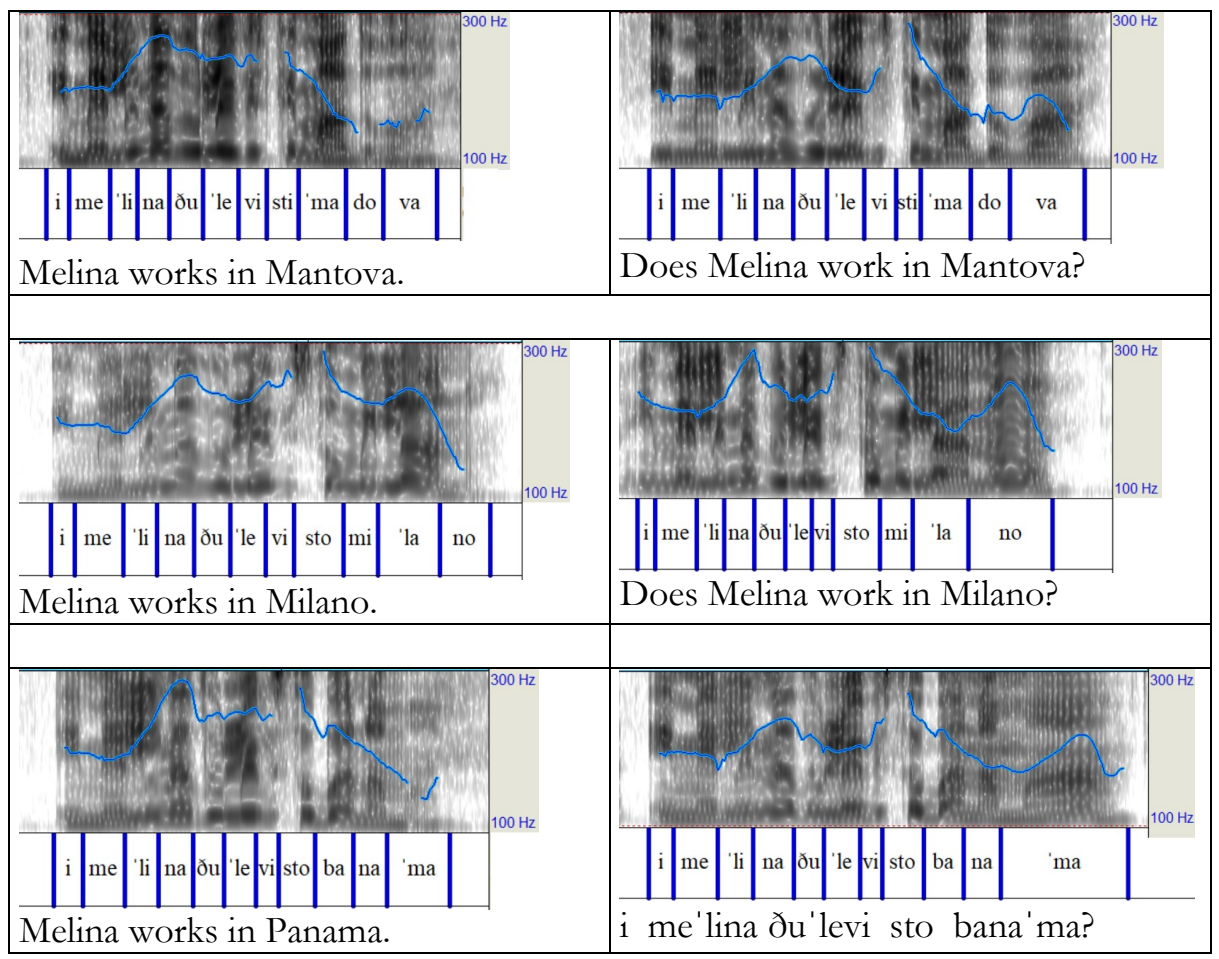

Figure 1. Tonal production of polylectic sentences as a function of lexical stress variability and statement (left) vs. question distinctions (right). 
Figure 2 shows the tonal production of statements (Figure 2, left column) and questions in one word examples (Figure 2, right column). Each word production has a different stress pattern associated with the final word, namely the stress is in the antepenultimate (upper panels), the penultimate (middle panels) and ultimate (lower panels).

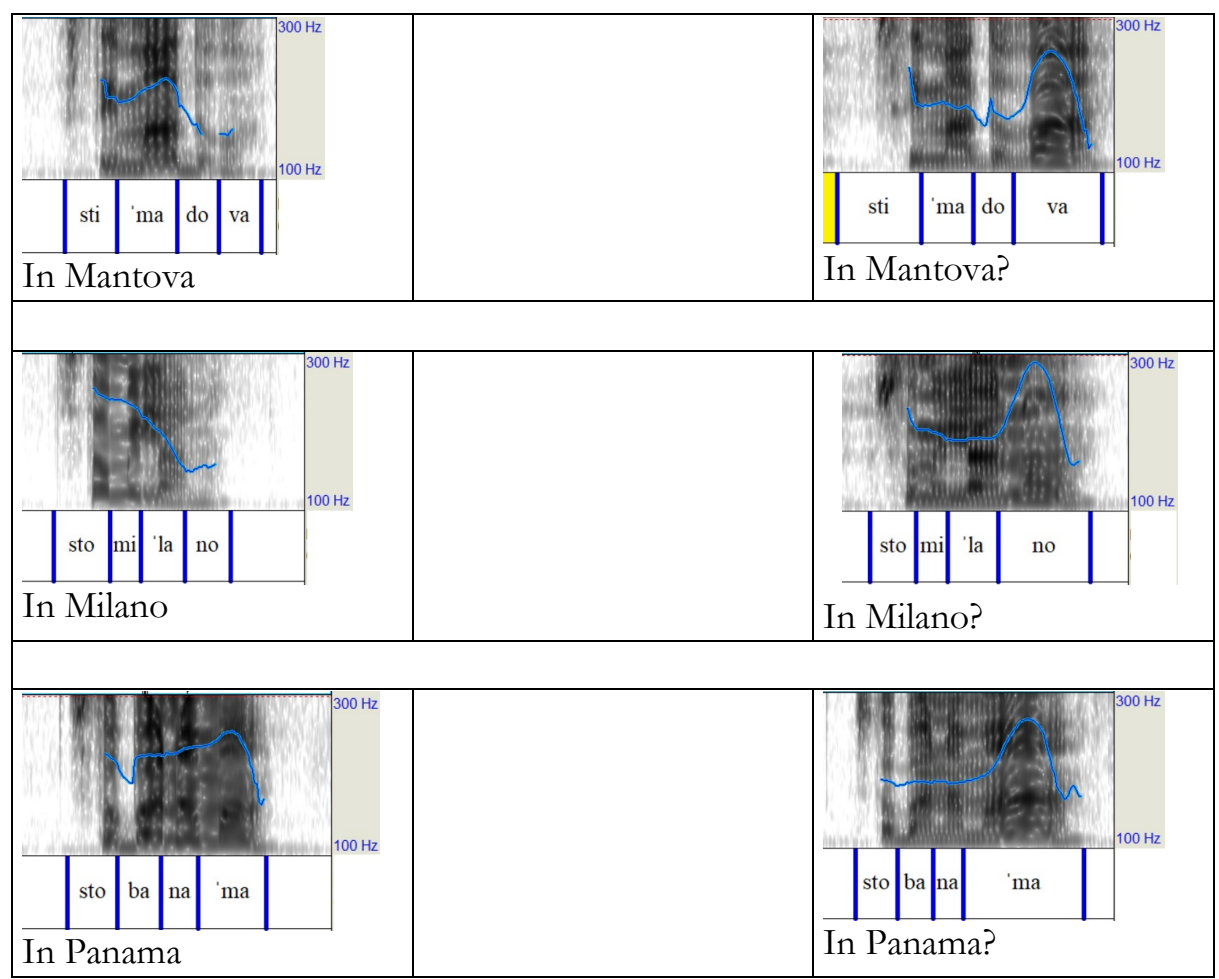

Figure 2. Tonal production of monolectic sentences as a function of lexical stress variability and statement (left) vs. question distinctions (right).

Statements and questions have different tonal patterns. The pattern for statements is a rise on the stressed syllable and a fall at the end of the utterance. This pattern is not evidenced due to constraints imposed by the positioning of the stress. For example, when the stress is on the ultimate syllable this fall does not take place. In questions the pattern is a lowering of the fundamental frequency on the stressed syllable and a rise. The lowering is not evidenced when the stress is on the ultimate syllable, since it coincites with the final rise. 


\section{Discussion}

The current study displays that Standar Greek like many other speech varieties exhibits language specific tonal patterns that characteristically distinguish Standard Greek from other language families such as Italian or Swedish and even other language varieties of Greek, such as Cypriot Greek (cf. Themistocleous 2011, 2014). On the other hand, it is related to earlier research in English that displays the distinct tonal patterns between statements and questions especially with the tones that demarcate phrase and sentence boundaries (Beckman, Edwards 1990).

The application of the final movements takes place primarily at the final syllables. In addition, this study demonstrates the effects of edge-tones and prosodic domain boundaries on final lengthening. Specifically, it shows that two types of tonality apply at the right edge of post-lexical domains: (a) preboundary tonal movement that marks the edges of prosodic domains and varies depending on boundary strength and (b) edge-tone lengthening that associates with the effects of syllable lengthening. The two types of tonal structure trigger different overlaying effects that account for the lengthening patterns at the edges of prosodic domains.

Beyond the results of the present study, our investigation has demonstrated a straightforward way for the elicitation of alternative questions in a questionquestion methodological paradigm. This methodology may be applied in a variety of languages with different prosodic and intonation systems, the results of which may be cross-linguistically and cross-dialectically compared in a direct way without any other linguistic variability but prosody.

\section{References}

Beckman, M., Edwards, J. 1990. Lengthenings and shortenings and the nature of prosodic constituency. In Kingston, J., Beckman, M. (Eds.), Papers in laboratory phonology I. Between the grammar and the physics of speech, 152-178. Cambridge University Press.

Botinis, Antonis. 1989. Stress and prosodic structure in Greek. Lund University Press.

Botinis, A., Chaida, A. Nikolaenkova, O., Nirgianaki, E. 2016. Intonation and polar questions in Greek frevisited. In Botinis, A. (Ed.), Proc. ExLing 2016, 41-44.

Themistocleous Ch. 2011. Prosodia kai plirophoriaki domi stin Ellinici (Prosody and Information Structure in Greek). PhD Thesis, University of Athens, Greece.

Themistocleous Ch. 2014. Edge-Tone Effects and Prosodic Domain Effects on Final Lengthening. Linguistic Variation 14(1). 129-160. DOI: 10.1075/lv.14.1.06the. 